\title{
Classification of Hip Shape and Customized Hip Augmentation in Asian Women
}

\author{
Woong Heo ${ }^{1}$, Bong Gweon Park ${ }^{2}$, \\ Seungki Youn ${ }^{1}$, Hee Chang Ahn ${ }^{1}$ \\ ${ }^{1}$ Department of Plastic and \\ Reconstructive Surgery, Hanyang \\ University Hospital, Hanyang University \\ College of Medicine, Seoul, ${ }^{2} \mathrm{~W}$ Plastic \\ Surgery, Seoul, Korea
}

Background We classified the hips of Korean women into 6 types based on shape and contour, and have performed gluteal augmentation using Elastomere implants with lipoplasty of the flank, gluteal fold, and saddle bags of the thigh according to the hip type.

Methods We analyzed the shapes and contour of the buttocks of 148 patients from September 2009 to September 2015. We performed buttock augmentation with implants using Gonzalez's XYZ method, as well as liposuction and fat injection lipoplasty of the surrounding areas in these 148 patients.

Results We defined the following 6 types: the oval type (30\%), the straight type (17\%), the peach type ( $9 \%)$, the trapezoid type (20\%), the heart type (11\%), and the W type (13\%). Ninety-seven patients underwent hip augmentation with an implant only or combined with lipoplasty, and 51 patients underwent lipoplasty for buttock shape correction. Patients were followed up for at least 3 months, with the longest follow-up being 4 years. Implants were removed postoperatively in 8 of 97 cases.

Conclusions The hip augmentation not only involves the simple insertion of implants, but also complex surgical procedures on the surrounding flank and thigh areas. Appropriate procedures should be utilized based on the 6 different hip types to ensure the best outcomes.

Keywords Buttocks, Female, Reconstructive surgical procedures, Surgical flaps

No potential conflict of inter
this article was reported.

\section{INTRODUCTION}

Humans first developed their hip muscles when they started to walk upright, and beautiful hips are known as a key feature of sexual appeal across cultures. Despite the long history of man's interest in hips, a scientific approach toward achieving beautifully shaped hips has only recently been explored.

The gluteus maximus muscles, which are crucial for walking, are often mobile and pressurized, and their use in hip augmentation

Received: Apr 19, 2017 Revised: Nov 29, 2017 Accepted: Dec 2, 2017 Correspondence: Hee Chang Ahn Department of Plastic and Reconstructive Surgery, Hanyang University Hospital, 222-1 Wangsimni-ro, Seongdong-gu, Seoul 04763, Korea. E-mail: ahnhc@hanyang.ac.kr

Copyright () 2018 The Korean Society for Aesthetic Plastic Surgery.

This is an Open Access article distributed under the terms of the Creative Commons Attribution Non-Commercial License (http://creativecommons.org/licenses/by-nc/4.0/) which permits unrestricted non-commercial use, distribution, and reproduction in any medium, provided the original work is properly cited. www.e-aaps.org surgery may result in a number of complications [1,2]. Hip-up surgery via implants was first reported by Robles et al. [3] in 1984, and went these procedures went through numerous iterations to reach the final methodology reported by Vergara and Marcoes [1] in 1996, which is still used at present. Implants are placed between the muscles in the hip-up operation, and the safety of this technique has been less studied than other surgical procedures, as it has been utilized for less than 20 years [4-6]. Caution ought to be taken in Korean women, as almost no practical research has been conducted in this population specifically. The hip muscles of Koreans are smaller and thinner than those of Caucasians, and implant insertion might therefore result in side effects or complications.

In this study, we classified the hips of Korean women into 6 types in an attempt to establish the distribution of various hip shapes and to approach them more safely. Surgery was carefully performed only after considering which type of hip a given patient had. Many other classifications can be considered, but these 6 types are thoroughly discussed and compared in this article. 
In terms of the surgical procedure, the gluteus maximus was dissected after its thickness was measured and its exact anatomical location was identified. We are confident that these hip operations are much safer and lead to more satisfactory outcomes if the gluteus maximus thickness is accurately measured via dissection between $\mathrm{X}$ and $\mathrm{Y}$ and about half of the gluteus maximus is dissected via the sandwich method. The continuous and accurate performance of the XYZ method described by Gonzalez [4,7] is crucial for ensuring patient safety and satisfaction.

\section{METHODS}

\section{Materials}

A total of 148 women underwent hip-up surgery with implants and body shape adjustment through liposuction from September 2009 to September 2015. Their hip shapes were categorized into 6 types and the surgical technique varied according to the type. The patients ranged in age from 19 to 59 years, with an average age of 39 years.

Forty-five patients exhibited the oval type, followed by 30 patients who were classified as having the trapezoid type. Twenty-five patients had the straight type, and the $\mathrm{W}$ and heart types accounted for 19 and 16 patients, respectively. Thirteen patients had hips belonging to the peach type. A total of 71 patients underwent hip-up surgery with implants only, and 26 patients had hip-up surgery with implants along with lipoplasty. Fifty-one patients underwent hip-up surgery with liposuction and injection (Table 1).

\section{Methods}

When dissecting the gluteus maximus, an incision was made perpendicularly to the muscle near the sacrum based on the XYZ method. Dissection was done near the higher inside point of the gluteus muscle, known as the $\mathrm{Y}$ point. The dissector was then rotated to the trochanter point, which is known as the $\mathrm{Z}$ point $[4,7]$. Too much dissection of the gluteus maximus can cause bleeding due to damage to surrounding vessel branches. Furthermore, a number of arteries run near the sacrum, and making an incision at the sacrum is not recommended. A smooth implant was inserted within the muscle, but a textured type was inserted beneath the muscle fascia. Use of textured implants in the muscle can be problematic when expanding the pocket, as the implant presses the pocket wall, which may result in a seroma.

If the implant is inserted too high, pain can result due to compressed cutaneous nerves in the pelvis, or it can cause a double buttock form. Similarly, if the implant is inserted too far below the pyramidal muscle, it can damage the sciatic nerve and inferior gluteal artery; this is why it is so important to dissect exactly half of the gluteus maximus.

\section{Classification of hip types}

Hips were classified based on the coronal view. Each type was defined based on the relative width of the waist line, iliac crest line, and greater trochanter line (Fig. 1).

\section{Oval type}

This type has a relatively narrow waist line and greater trochanter line, in contrast to a wide iliac crest line. This is the most common type, and satisfactory surgical results can be achieved in these patients if the center of the hips is raised, along with filling of the coxae parts (femur area) sideways in hip-up surgery with implants. Round implants are recommended for better results, based on fact that the sides of the pelvis in most Asians are oval. The ideal position of the implant is approximately 1.0 to $1.5 \mathrm{~cm}$ upward from the center of the hips, which pulls up the gluteus maximus and the drooped fat on the sides and makes the legs look longer.

\section{Straight type}

Patients with the straight type have straight lines along their waist, pelvis, and thighs, and this is often due to a lack of volume in the hips. Oval types and straight types are distinguished by the fact that the pelvis in the former type is round and the hips are seemingly stereoscopic, whereas the pelvis in the latter type is relatively longer lengthways and the hips are flat. The preferable implant is generally oval-shaped; however, as Koreans have small pelvises, this may result in some discomfort in the hips when seated; therefore, we used round implants. The insertion is best done slightly

Table 1. Summary of patient data

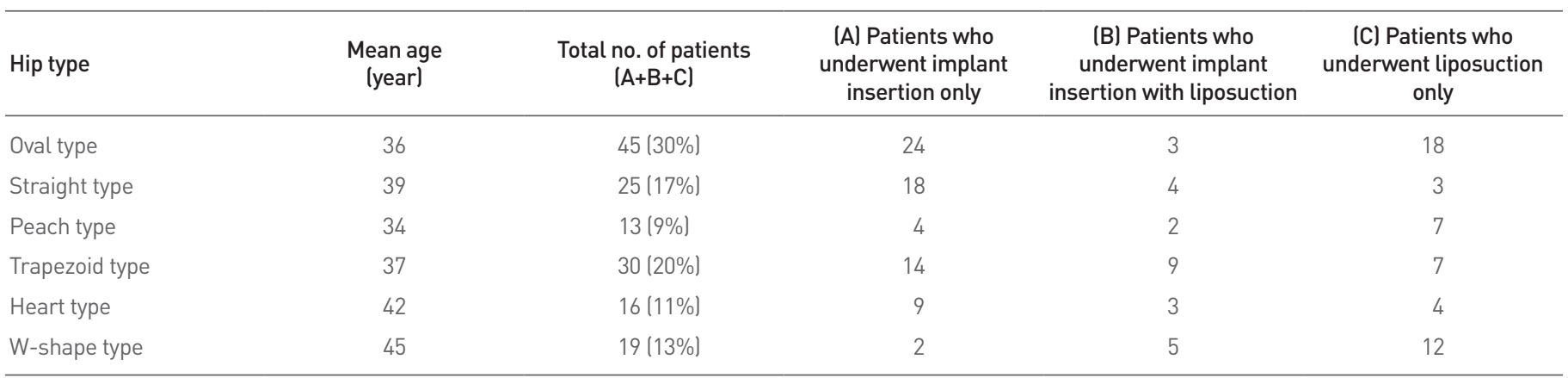



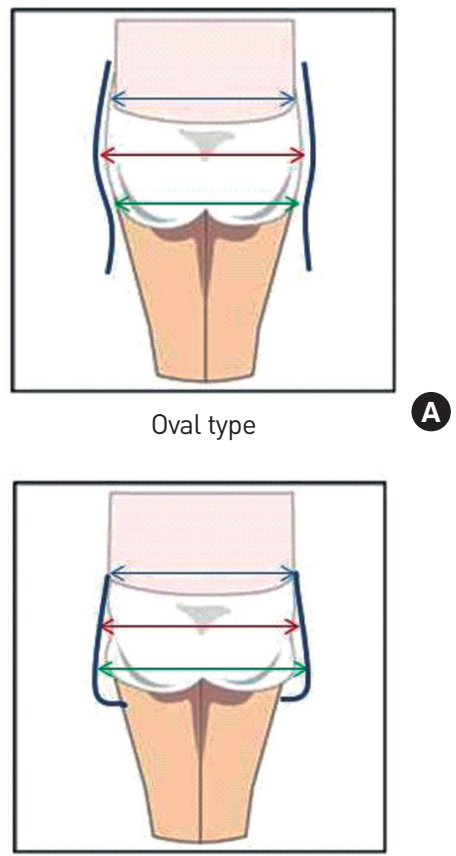

Trapezoid type
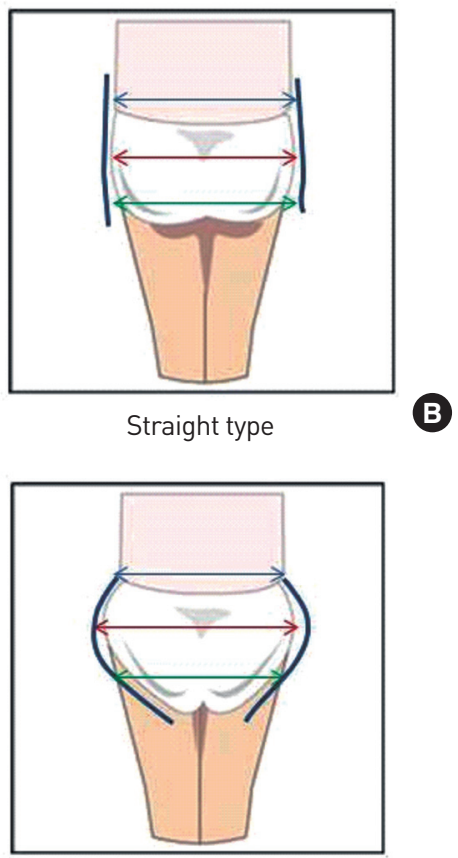

Heart type
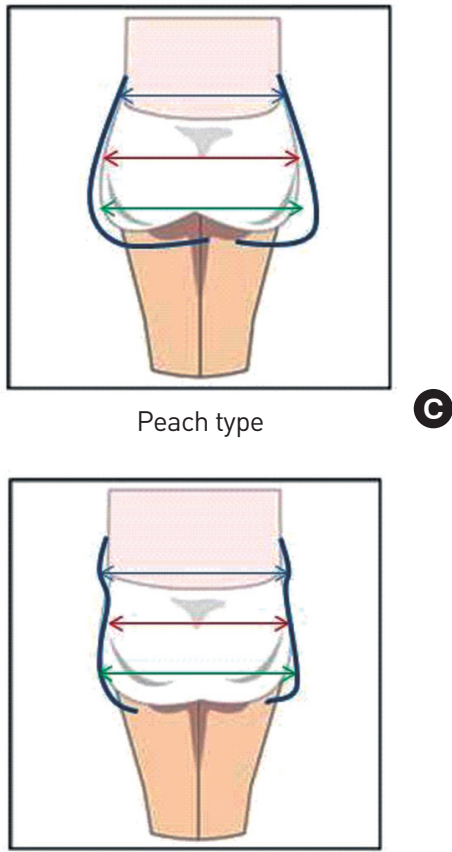

W-shape type

Fig. 1. Hip types were classified based on the coronal view of the hips. Each type was characterized based on the relative width of the waist line, iliac crest line, and greater trochanter line. (A) The oval type has a relatively narrow waist line and great trochanter line, in contrast to a wide iliac crest line. (B) Patients with the straight type have straight lines along their waist, pelvis, and thighs. (C) The peach type refers to a slim waist and iliac crest line with stout thighs. (D) The trapezoid type is characterized by a straight line along the waist and hips, while the sides of the thighs are highly projected. (E) In the heart type, a great deal of fat is located on the waist, and in many cases the pelvis appears to be shaped like a heart. (F) The W type has the waist and thighs projected sideways with a relatively narrow iliac crest line.

higher than the center of the hips. Additionally, liposuction of the waist is recommended to create a more feminine body line, and the thighs can also be adjusted with liposuction if necessary. Implants are the best way to achieve satisfaction in patients with this type of hip, because they alter the form of the flat hips. Liposuction and fat injection alone are less likely to satisfy patients with this hip type.

\section{Peach type}

This type refers to a slim waist and iliac crest line with stout thighs, and the iliac crest is sometimes as wide as the intertrochanteric distance. If hip-up surgery with an implant and liposuction on the sides of the thighs and fat injection on the coxae are successfully performed, natural-looking, glamorous hips will result. The underside crease line of this type is drooped overall, and it is for this reason that adjusting this line is a difficult task. If a patient is prone to scarring fairly easily or if the line of the hip crease is drooped itself, lifting surgery would instead be recommended rather than an implant. Consultation with plastic surgery specialists is crucial because Asians are prone to scarring.

\section{Trapezoid type}

This type is characterized by a straight line along the waist and hips while the sides of the thighs are highly projected. The distance at the waist is the least wide, followed by the distances at the iliac crest and trochanters, in order. Liposuction of the sides of the thighs and hip-up surgery with implants will lead to satisfactory results; however, if implants are deemed too risky, centrifugation after fat harvest on the waist and thighs with subsequent fat injection can also be an option.

Individuals with the peach type have a waistline that is slimmer than the pelvis. When viewed from the side, the line along the waist and hips makes a slightly drooping curve. In contrast, the trapezoid type has a straight line from the waist to the hips. When looked at from the side, the body line is almost straight. In other words, the trapezoid type can be considered a variation on the straight type, with a large amount of fat in the upper thighs. Both types involve either flat or drooped hips, so that implants are necessary to achieve a beautiful hip. Patients with the trapezoid type also require liposuction, whereas it is optional for patients with the peach type.

\section{Heart type}

Because a great deal of fat is located around the waist, the pelvis appears to be shaped like a heart in many cases. The distance between the iliac crest is the widest due to this pattern of fat deposition. Liposuction should be performed to remove the fat, and implants should be inserted in the exact center to achieve a natural hip line 
and desirable body line. This type is often seen in women over age 40 whose thigh fat and drooped hips shrink and settle on the abdomen and flank, making the ilium line wider. In these patients, liposuction of the flanks is absolutely necessary, with optional body line adjustment. However, if the inverted triangular shape is formed due to the shape of the pelvic bone, further consultation is required based on the patient's needs and wants.

\section{W type}

In this type, the waist and thighs are projected sideways with a relatively narrow iliac crest line. Liposuction of the waist and thighs along with hip-up surgery with implants is recommended. This type is most frequently observed in stout young females, and tends to be associated with the least satisfaction. Since there is ample fat to be harvested, the fundamental aim of the operation is more body line adjustment through liposuction rather than hip-up surgery. However, in cases with flat hips, hip-up surgery with implants can be used. Liposuction beneath the center of the hips is strongly discouraged in terms of patient satisfaction. Any harvested fat should be injected based on the body shape and form.

The heart types and $\mathrm{W}$ types are often present in older women. Their waistlines are overall wider than their pelvic line and their pelvic line is vague (sunken). The $\mathrm{W}$ types is often the result of weight gain and changes in body shape due to aging, and these patients are more likely to need liposuction than those with the heart type. Heart-type hips are often flat, whereas $\mathrm{W}$ type hips have fat beneath the center of the hips, like the peach type. When surgery is to be performed, it is recommended that both the heart and $\mathrm{W}$ types undergo liposuction first. In patients with trapezoid-type hips, hip-up surgery and liposuction frequently take place at the same time. For patients with oval-type, straight-type, and peachtype hips, hip-up surgery should take place prior to liposuction for the best outcomes.

\section{RESULTS}

Only 8 patients had their implants removed: 3 due to infection, another 3 due to postoperative pain, 1 due to bleeding, and 1 due to dissatisfaction. The remaining operations with implants, liposuction, and fat injection were successful, and the patients were satisfied with the results.

Hip-up surgery was performed according to the hip type. Specific examples are described below.

\section{Case study 1. Oval type (Fig. 2)}

A 26-year-old woman had a hip size of $84 \mathrm{~cm}$ and a height of 156 $\mathrm{cm}$, with a short, round pelvis, and her hips were projected compared to her body size. She had a body line including a relatively narrow waist line and great trochanter line, in contrast to a wide iliac crest line. A round Elastomere bag $(250 \mathrm{~mL})$ was inserted. She
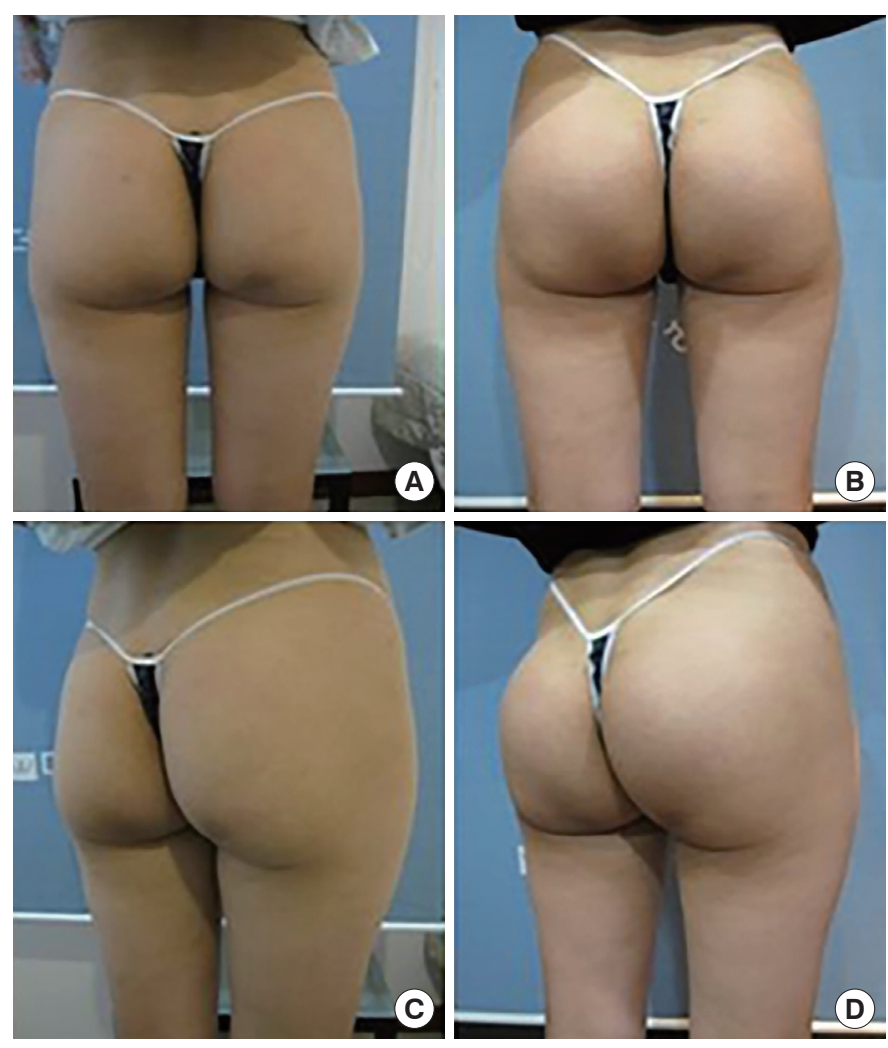

Fig. 2. Case 1 (oval type). A 26-year-old woman had a hip size of 84 $\mathrm{cm}$ and a height of $156 \mathrm{~cm}$, with a short, round pelvis, and her hips were projected compared to her body size. She had a body line including a relatively narrow waist line and great trochanter line, in contrast to a wide iliac crest line. A round Elastomere bag $(250 \mathrm{~mL})$ was inserted. She was followed for 6 months postoperatively, and her body line was corrected; more volume was shifted to the hips and the sides of the hips were lifted. (A) Preoperative posterior view. (B) Postoperative posterior view. (C) Preoperative oblique view. (D) Postoperative oblique view.

was followed for 6 months postoperatively, and her body line was corrected; more volume was shifted to the hips and the sides of the hips were lifted.

\section{Case study 2. Straight type (Fig. 3)}

A 31-year-old woman with a hip size of $81 \mathrm{~cm}$ and a height of 168 $\mathrm{cm}$ had flat hips and a straight body line along the waist, pelvis, and thighs. She underwent insertion of a round Elastomere implant $(200 \mathrm{~mL})$ with liposuction of her waist and thighs. Her hips were lifted and had become more voluminous at 3 months postoperatively.

\section{Case study 3. Peach type (Fig. 4)}

A 36-year-old woman with a hip size of $83 \mathrm{~cm}$ had a right hip that was smaller than the left. She had a slim waist and iliac crest line with stout thighs, and underwent insertion of a round Elastomere 

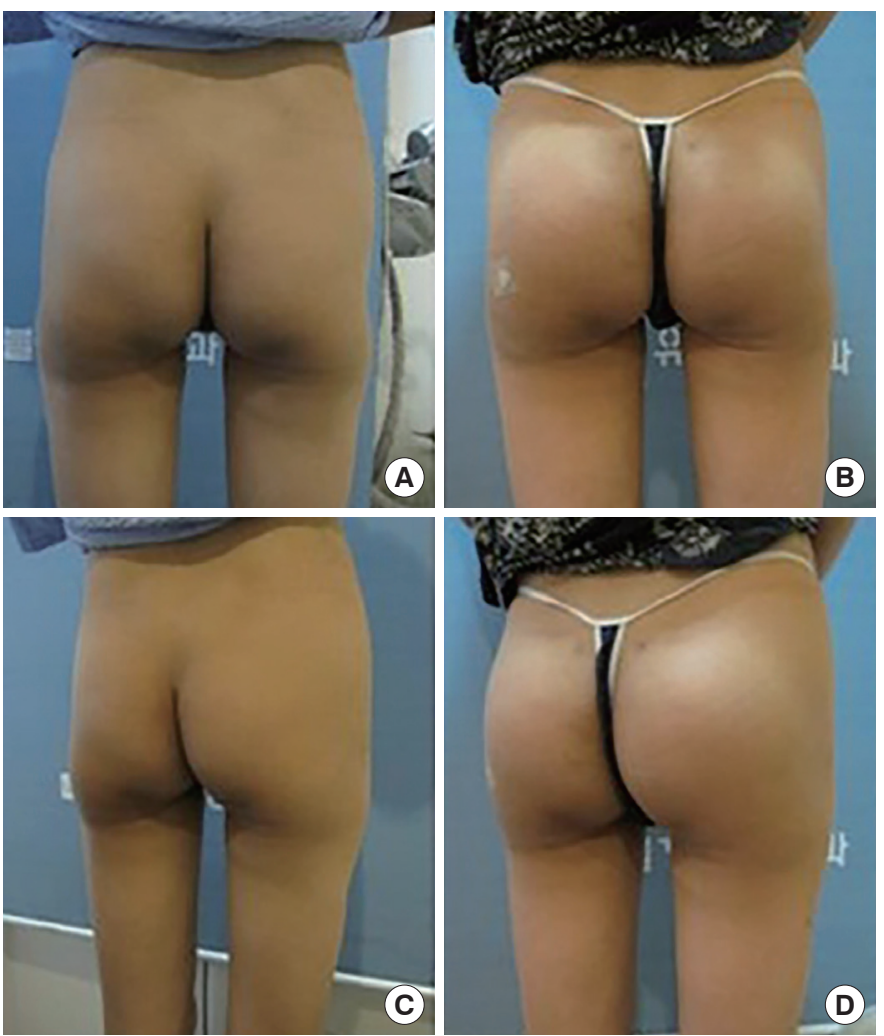

Fig. 3. Case 2 (straight type). A 31-year-old woman with a hip size of $81 \mathrm{~cm}$ and a height of $168 \mathrm{~cm}$ had flat hips and a straight body line along the waist, pelvis, and thighs. She underwent insertion of a round Elastomere implant ( $200 \mathrm{~mL}$ ) with liposuction of her waist and thighs. Her hips were lifted and had become more voluminous at 3 months postoperatively. (A) Preoperative posterior view. (B) Postoperative posterior view. (C) Preoperative oblique view. (D) Postoperative oblique view.

implant $(250 \mathrm{~mL})$. More tension was applied to the right gluteus maximus than the left during suturing. A year postoperatively, her hip shape had become more natural than before.

\section{Case study 4. Trapezoid type (Fig. 5)}

A 32-year-old woman had a hip size of $87 \mathrm{~cm}$, as well as flat hips and a considerable amount of fat on the outer parts of her thighs. She had a straight line along the waist and hips, while the sides of the thighs were projected. She underwent insertion of a round Elastomere implant $(250 \mathrm{~mL})$ and liposuction of the thighs.

\section{Case study 5. Heart type (Fig. 6)}

A 43-year-old woman with a fatty waist had $850 \mathrm{~mL}$ liposuctioned from her waistline and $500 \mathrm{~mL}$ from her thighs. The harvested fat was centrifuged at 4,000 rpm for 10 minutes. An injection of 250 $\mathrm{mL}$ of pure fat was made into each hip-up line and the gluteus maximus. This patient ultimately wanted more volume, so hip-up surgery with an implant $(200 \mathrm{~mL})$ was performed 3 months later.
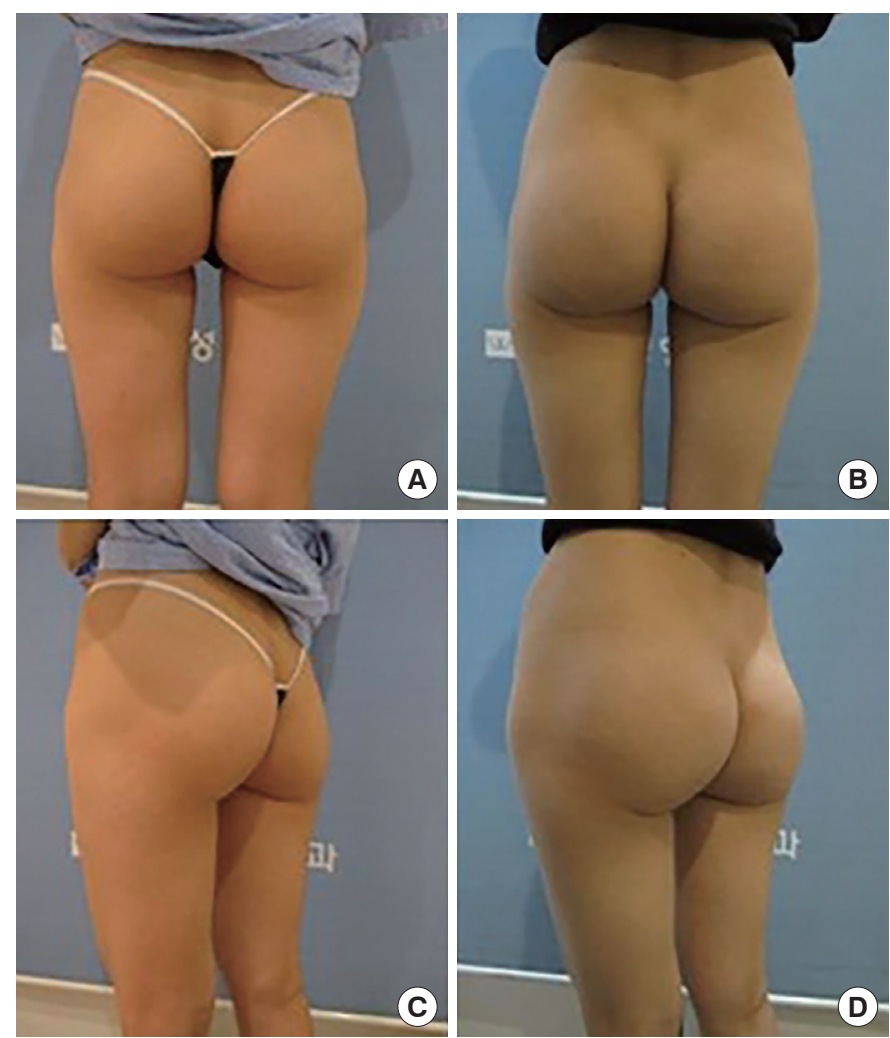

Fig. 4. Case 3 (peach type). A 36-year-old woman with a hip size of $83 \mathrm{~cm}$ had a right hip that was smaller than the left. She had a slim waist and iliac crest line with stout thighs, and underwent insertion of a round Elastomere implant ( $250 \mathrm{~mL}$ ). More tension was applied to the right gluteus maximus than the left during suturing. A year postoperatively, her hip shape had become more natural than before. (A) Preoperative posterior view. (B) Postoperative posterior view. (C) Preoperative oblique view. (D) Postoperative oblique view.

\section{Case study 6. W type (Fig. 7)}

A 29-year-old woman with a hip size of $91 \mathrm{~cm}$ and height of 165 $\mathrm{cm}$ had waist and thighs that projected sideways. She underwent insertion of a round 290-mL implant and liposuction of her flanks and thighs. Forty months after surgery, her overall body shape was observed to be more glamorous and she was highly satisfied with her body.

\section{DISCUSSION}

Hip-up surgery with implants is technically difficult and likely to fail without accurate dissection of the gluteus maximus $[4,7,8]$. A lack of confidence and experience can lead to postoperative problems such as pain or bleeding, and these problems tend to recur [8]. Accuracy is essential in the dissection of the gluteus maximus and insertion of implants. In patients with peach-type hips, the upper gluteus is thin, so the implant ought to be inserted deeper down; otherwise, the implant can be seen on the surface of the skin after 

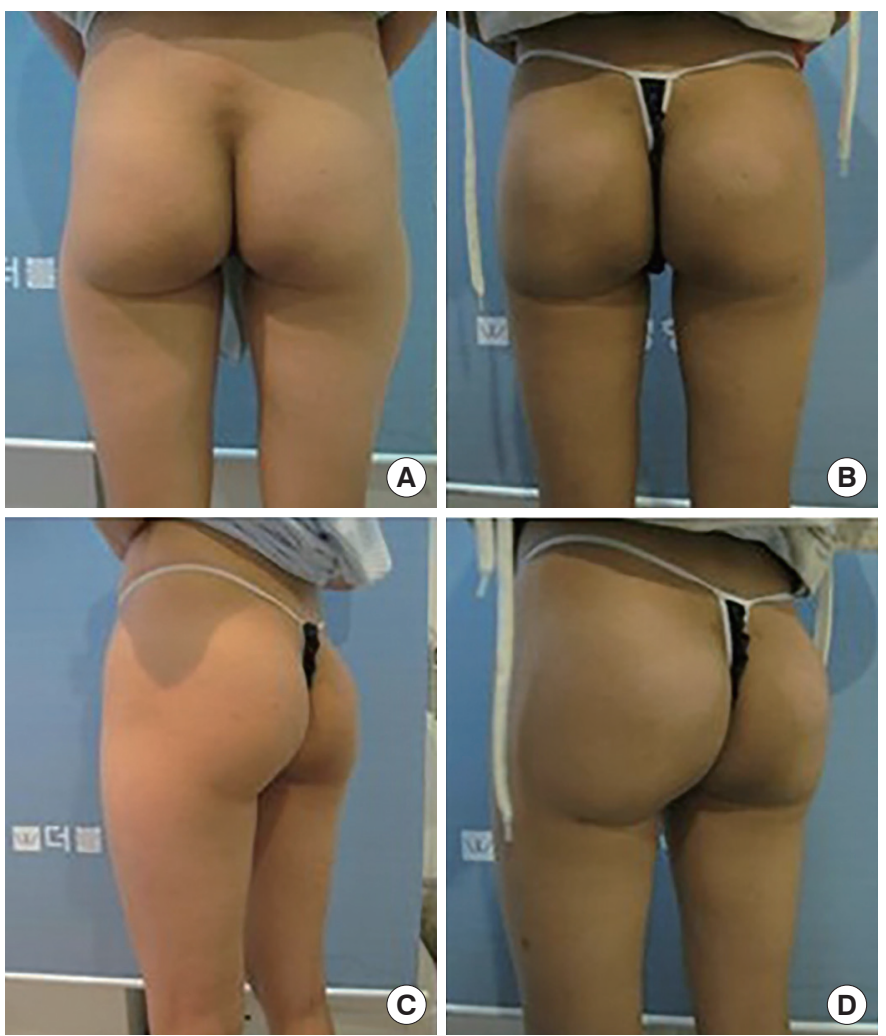

Fig. 5. Case 4 (trapezoid type). A 32-year-old woman had a hip size of $87 \mathrm{~cm}$, as well as flat hips and a considerable amount of fat on the outer parts of her thighs. She had a straight line along the waist and hips, while the sides of the thighs were projected. She underwent insertion of a round Elastomere implant $(250 \mathrm{~mL})$ and liposuction of the thighs. (A) Preoperative posterior view. (B) Postoperative posterior view. (C) Preoperative oblique view. (D) Postoperative oblique view.

surgery. For trapezoid types in which liposuction and implant surgery take place at the same time, it is possible to harvest the fat in protruded area and then inject it into depessed area at the same time of implant insertion. The center of the pelvis is located lower than the center of the hips, so the legs look shorter than they actually are. In order to overcome this, implants should be inserted slightly higher than the center of the hips. Postoperative follow-up is as important as the surgical procedure itself. Patients in this cohort were followed for a maximum of 4 years. All potential problems with this procedure should be addressed via continuous follow-up and observation.

The key element distinguishing the hip types is the form of the pelvis, because it is the primary contributor to the shape of the hips, and the shape varies further depending on how much fat is present and where it is located. This is also where dissatisfaction arises. Liposuction and fat injection, in particular, can never be the sole solution for augmenting hip volume even if the body shape can be satisfactorily adjusted [9]. Hip-up surgery with implants, in con-
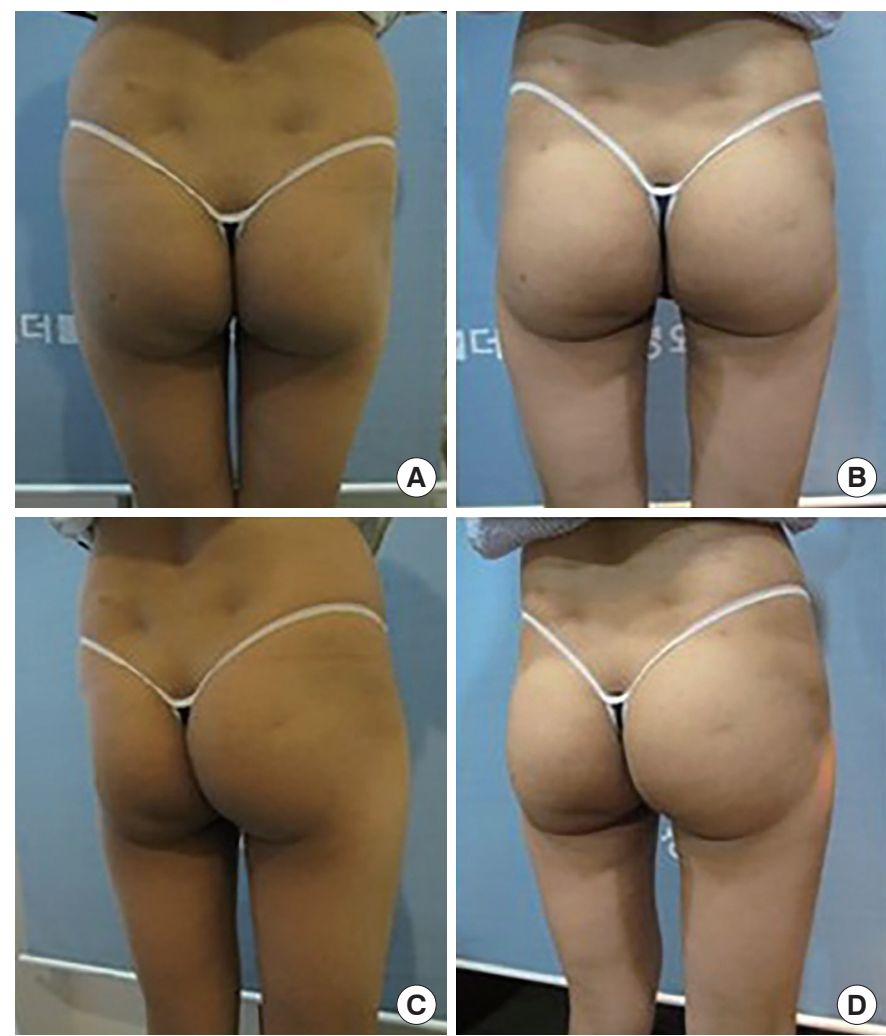

Fig. 6. Case 5 (heart type). A 43-year-old woman with a fatty waist had $850 \mathrm{~mL}$ liposuctioned from her waistline and $500 \mathrm{~mL}$ from her thighs. The harvested fat was centrifuged at 4,000 rpm for $10 \mathrm{~min}-$ utes. An injection of $250 \mathrm{~mL}$ of pure fat was made into each hip-up line and the gluteus maximus. This patient ultimately wanted more volume, so hip-up surgery with an implant ( $200 \mathrm{~mL}$ ) was performed 3 months later. (A) Preoperative posterior view. (B) Postoperative posterior view. (C) Preoperative oblique view. (D) Postoperative oblique view.

trast, is a definitive solution for changing the shape of the hip, and the key to successful surgery is an anatomical understanding of the gluteus maximus.

The gluteus maximus, the major muscle of the hips, has significant adjacent muscles and tissues, and a thorough understanding of the anatomy reduces the surgical difficulties. A proper understanding includes the neural distribution capable of causing pain, the main muscles around the gluteus maximus, and the vasculature.

The size of the gluteus maximus is typically $24 \times 24-\mathrm{cm}$ and its thickness is approximately 3 to $4 \mathrm{~cm}$. It is a hematogenous type III muscle with dual blood supply. The blood supply runs through the internal iliac artery upward from the piriformis muscle [10]. The superior gluteal artery and inferior gluteal artery are split internally; the upper side of the superior gluteal artery makes its way to the tensor fascia lata and the anterior superior iliac spine across the gluteus minimus, whereas the bottom proceeds to the greater trochanter of the femur. It circulates blood in the gluteus maximus 

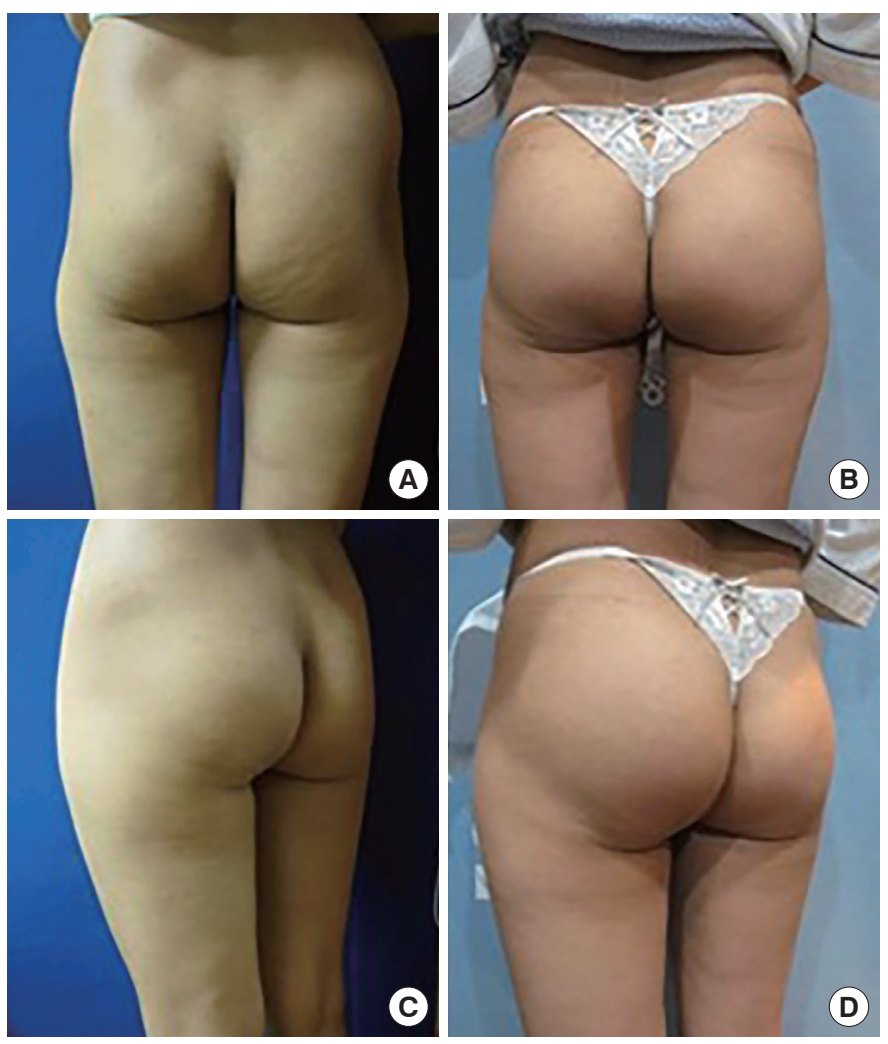

Fig. 7. Case 6 (W type). A 29-year-old woman with a hip size of 91 $\mathrm{cm}$ and height of $165 \mathrm{~cm}$ had waist and thighs that projected sideways. She underwent insertion of a round $290 \mathrm{~mL}$ implant and liposuction of her flanks and thighs. Forty months after surgery, her overall body shape was observed to be more glamorous and she was highly satisfied with her body. (A) Preoperative posterior view. (B) Postoperative posterior view. (C) Preoperative oblique view. (D) Postoperative oblique view.

and hip joints. The inferior gluteal artery goes beneath the piriformis muscle and towards the gluteus maximus and the ischial tuberosity. It is long and thin, proceeds to the lower side of the thighs, and is mainly responsible for the blood circulation of the pelvic limbs $[1,11,12]$.

The inferior gluteal nerve is rooted in the gluteus maximus and originates from L5, S1, and S2. There is also a cutaneous nerve that originates from the lumbosacral and third sacral nerve in the waist. This area is also where postoperative pain originates, as swelling after surgery can compress these nerves [8]. This is important because it is especially difficult to secure a safe place for the implants in Koreans who have small pelvises and therefore smaller gluteus maximus muscles. The specific size can be determined once the gluteus and pyramid muscles are identified. The pyramid muscles originate from the sacrum and insert at the greater trochanter. The superior gluteal artery, vein, and nerve run above this muscle, and below that run the sciatic nerve; the inferior gluteal artery, vein, and nerve; the pudendal nerve; and the cutaneous nerve [13]. In short, the lower edge of the pyramid muscles is the boundary for the implant. If the implant is not appropriately inserted in this limited space, pain due to bent implants or dissatisfaction due to stretched ligaments can occur after surgery. This procedure is important if the pubis is projected due to angulation between the pubis and the gluteus maximus, necessitating that the gluteus maximus be dissected more deeply. In Korean women, implants can be inserted suprafascially due to the danger of atrophy of the gluteus maximus.

The muscles between the hips are thick at their origins, but the gluteus maximus is thinner than it seems. Thus, experience in dissection and careful observations are required $[1,14]$. The choice between implants and fat injection should be made carefully based on the patient's desires. If the thickness of the gluteus maximus is accurately measured through dissection between $\mathrm{X}$ and $\mathrm{Y}$ and about half of the gluteus maximus is dissected using the sandwich method, satisfactory results are almost guaranteed. More satisfactory hip-up surgery with implants by the XYZ method can be achieved with lipoplasty according to the patient's hip type [4,7].

Round implants are preferable for oval-type hips. An oval implant with liposuction of the waist is recommended for the straight type. In the peach type, lifting surgery is recommended rather than an implant. The trapezoid type has either flat or drooped hips, and the insertion of round implants with liposuction is preferred. In the heart type, liposuction of the flank typically is absolutely necessary. In the $\mathrm{W}$ type, liposuction beneath the center of the hips is highly discouraged, and any harvested fat should be injected based on the body shape and form.

\section{CONCLUSIONS}

The desire for beautiful hips is strong among Korean women. Those with small, flat hips regard hip-up surgery as a potential solution. Hip-up surgery with implants not only alters the shape of the hips, but can contribute to a patient's satisfaction and higher self-esteem. For successful hip-up surgery, in this study we categorized hip shape into 6 types in an attempt to distinguish between the required surgical method and approaches. We made suggestions regarding implant size and shape and the implant insertion procedure for obtaining satisfactory outcomes. Liposuction and lipoinjection are needed for better aesthetic outcomes [15].

The choice between using implants or fat injection in hip-up surgery should be made according to the advantages of each technique, and they both play a role in body shape adjustment to meet patient desires. However, procedures with implants are particularly outstanding in that they look very natural and the outcomes are long-lasting. Additional research is needed to further reduce their possible complications. 


\section{PATIENT CONSENT}

Patients provided written consent for the use of their images.

\section{REFERENCES}

1. Vergara R, Marcos M. Intramuscular gluteal implants. Aesthetic Plast Surg 1996;20:259-62.

2. Ford RD, Simpson WD. Massive extravasation of traumatically ruptured buttock silicone prosthesis. Ann Plast Surg 1992;29:86-8.

3. Robles JM, Tagliapietra JL, Grandi MA. Gluteoplastia de aumento: implante submuscular. Cir Plast Iberolatinoamer 1984;10:365-75.

4. Gonzalez R. Augmentation gluteoplasty: the XYZ method. Aesthetic Plast Surg 2004;28:417-25.

5. Mendieta CG. Gluteoplasty. Aesthet Surg J 2003;23:441-55.

6. González-Ulloa M. Gluteoplasty: a ten-year report. Aesthetic Plast Surg 1991;15:85-91.

7. Gonzalez R. Gluteal implants: the "XYZ" intramuscular method. Aesthet Surg J 2010;30:256-64.
8. Serra F, Aboudib JH, Cedrola JP, et al. Gluteoplasty: anatomic basis and technique. Aesthet Surg J 2010;30:579-92.

9. Centeno RF. Gluteal aesthetic unit classification: a tool to improve outcomes in body contouring. Aesthet Surg J 2006;26:200-8.

10. Mathes SJ, Nahai F. Clinical applications for muscle and musculocutaneous flaps. St. Louis, MO: Mosby; 1982.

11. Akita K, Sakamoto H, Sato T. Arrangement and innervation of the glutei medius and minimus and the piriformis: a morphological analysis. Anat Rec 1994;238:125-30.

12. Harrison D, Selvaggi G. Gluteal augmentation surgery: indications and surgical management. J Plast Reconstr Aesthet Surg 2007;60:922-8.

13. Jacobs LG, Buxton RA. The course of the superior gluteal nerve in the lateral approach to the hip. J Bone Joint Surg Am 1989;71:1239-43.

14. Perén PA, Gómez JB, Guerrerosantos J, et al. Gluteus augmentation with fat grafting. Aesthetic Plast Surg 2000;24:412-7.

15. Cárdenas-Camarena L, Lacouture AM, Tobar-Losada A. Combined gluteoplasty: liposuction and lipoinjection. Plast Reconstr Surg 1999; 104:1524-31. 